\title{
USO DE LAS PLANTAS MEDICINALES DEL DISTRITO DE QUERO, JAUJA, REGIÓN JUNÍN, PERÚ
}

\section{USE OF MEDICINAL PLANTS FROM THE DISTRICT OF QUERO, JAUJA, JUNÍN REGION, PERU}

\author{
Gladys Tello-Ceron ${ }^{1}$, Mercedes Flores Pimentel ${ }^{2}$ y Vilma Gómez Galarza ${ }^{2}$
}

\begin{abstract}
Resumen
Se llevó a cabo un estudio etnobotánico en la comunidad de Quero, ubicada en el Distrito de Molinos, Provincia de Jauja, Región Junín, Perú, con el fin de documentar las prácticas ancestrales asociadas al uso de plantas medicinales. Como resultado de este estudio se encontró un total de 62 especies agrupadas en 47 géneros y 28 familias. Las familias con mayor número de especies utilizadas fueron Asteraceae, Geraniaceae y Urticaceae; de acuerdo a su uso, las especies fueron agrupadas en 12 categorías de dolencias y 37 sub-categorías, siendo algunos: traumatismos, afecciones respiratorias, dolencias no definidas (susto, colerina, etc.) y digestivas, las más recurrentes. Por lo general las plantas se utilizan enteras, pero también se usan por separado las hojas y flores.
\end{abstract}

Palabras clave: etnobotánica, Andes, naturistas, Perú, puna, bola de nieve.

\begin{abstract}
An ethnobotanical study was carried out in the community of Quero, located in the District of Molinos, Province of Jauja, Junin Region, Peru. This research was aimed in documenting the use of ancestral practices regarding the use of medicinal plants. A total of 62 species grouped into 47 genera and 28 families were found. The families with the highest number of used medicinal species were Asteraceae, Geraniaceae and Urticaceae. According to their use, the species were grouped into 12 disease categories and 37 sub-categories, being traumatism, respiratory diseases, undefined ailments (fright, cholerine, etc.) and digestive disorders the most recurrent. The entire plant is commonly used, but leaves and flowers are also used separately.
\end{abstract}

Key words: ethnobotany, Andes, naturists, Peru, puna, snowball.

\section{Introducción}

Investigadores que desarrollan estudios en diferentes partes del mundo señalan que el conocimiento y uso de plantas medicinales se incrementa en relación directa con la cercanía de las personas que las aprovechan (Sõukand \& Kalle, 2010; Pardo de Santayana et al., 2011). Según la Oficina Regional de la Organización Mundial de la Salud para las Américas (AMRO / OPS), países como Chile y Colombia muestran que sus poblaciones utilizan la medicina tradicional en $71 \%$ y $40 \%$ respectivamente (Revene et al., 2008) y en la India el $65 \%$ de la población rural utiliza las plantas medicinales para atender sus necesidades primarias de salud. Asimismo, "los países desarrollados están popularizando los medicamentos tradicionales, complementarios y alternativos. Por ejemplo, el porcentaje de la población que ha utilizado dichos medicamentos al menos una vez es del $48 \%$ en Australia, el $31 \%$ en Bélgica, el $70 \%$ en el Canadá, el $42 \%$ en los Estados Unidos de América y el 49\% en Francia" (OMS, 2003).
Perú tiene una amplia riqueza de plantas medicinales con más de 4000 especies de usos conocidos por las poblaciones locales, de las cuales un gran porcentaje se presenta en la región andina (Brack, 1999; De la Cruz et al., 2007; Weckerle et al., 2013). El uso de plantas medicinales incluye el conocimiento, uso y manejo de una elevada riqueza de especies a través de complejas formas de interacción entre las comunidades locales y su entorno, información que se manejaba mucho antes de la llegada de la medicina occidental y la tecnología informática (Caballero et al., 2004; De la Torre et al., 2006; Bussmann et al., 2007a).

Sin embargo, en estos últimos años, ha ocurrido una pérdida importante del conocimiento tradicional del uso de las plantas; asimismo, la disponibilidad de las especies medicinales se ha visto reducida por la degradación de los ambientes naturales (Schultes, 1997; Bermúdez et al., 2005; Malengreau, 2007; Bussmann et al., 2007b), peligrando dicha relación.

Más aún, diversos estudios, sobre el estado de la investigación etnobotánica en los Andes del Perú, reportan que la mayoría de los trabajos etnobotánicos 


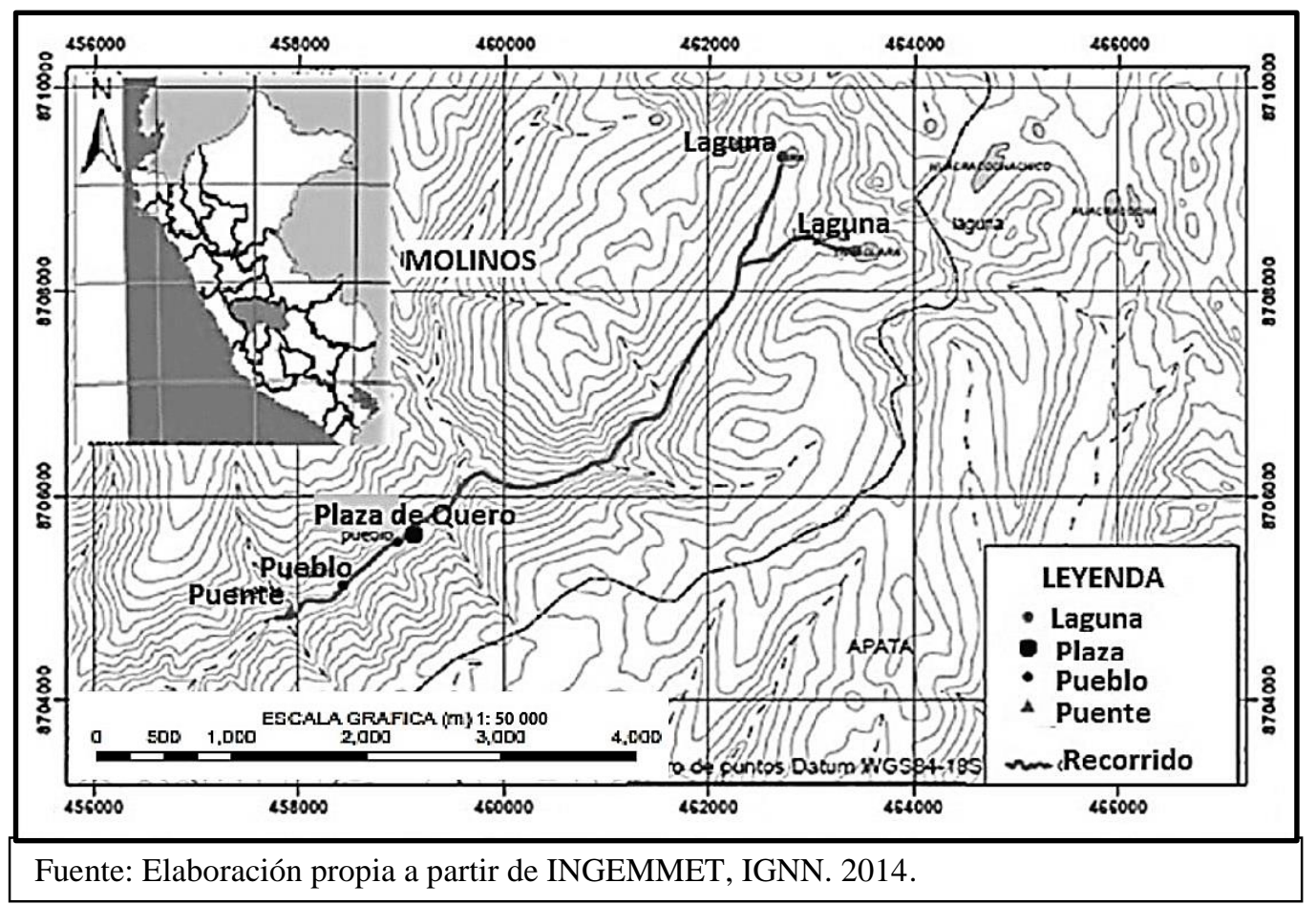

Figura 1. Mapa de la comunidad de Quero.

centran en los pisos de vegetación entre 1500 y 2500 metros de elevación, siendo los estudios en el Callejón de Huaylas (Áncash) los que abarcan mayor rango de altitud (3 000 - 5000 metros de elevación; (La TorreCuadros \& Albán, 2006). Los estudios etnobotánicos en los Andes peruanos dan a conocer la importancia del uso de las plantas medicinales en comunidades rurales, identifican a sus pobladores como transmisores del vasto conocimiento ancestral, así como el respeto por la naturaleza que le brinda salud y bienestar a sus vidas (Horák et al., 2015).

El distrito de Quero de la provincia de Jauja presenta diversos pisos térmicos que van desde los 3900 a los 4 800 metros de altitud, entre los cuales se distribuye una rica flora nativa, resaltando las plantas medicinales cuyas propiedades son conocidas, y por lo tanto utilizadas, para el tratamiento de enfermedades comunes; además, Siura \& Flores (2010) registraron un total de 42 especies medicinales para este lugar. Por ello, esta investigación tiene como objetivo documentar los conocimientos en el uso de las plantas medicinales importantes para el poblador.

\section{Materiales y métodos}

Área de estudio

La comunidad de Quero se ubica en el Distrito de Molinos, Provincia de Jauja, Región Junín, Perú (Figura 1), sus coordenadas son $11^{\circ} 40^{\prime}$ latitud sur y $75^{\circ} 20^{\prime}$ longitud oeste. Sus pobladores tienen como principales actividades la agricultura, ganadería, recolección y venta de plantas con uso medicinal en el mercado de Jauja (Gómez \& Vilca, 2006).
Metodología

Se realizaron entrevistas semiestructuradas (apéndice 1) a un total de 23 personas mayores de 35 años, consideradas como recurrentes en el uso de las plantas medicinales (Alexiades, 1996; De-la-Cruz et al., 2007; Blasco \& Otero, 2008; Ceuterick et al., 2011; Odonne et al., 2013; Albuquerque et al., 2015; Zeni et al., 2015), y con la técnica "bola de nieve" (Martínez, 2011; Zeni et al., 2015) se consiguió, adicionalmente, el nombre de los "naturistas" personas conocedoras del uso de las plantas medicinales.

Asimismo, se hicieron caminatas etnobotánicas junto a tres "naturistas" y se colectaron las especies medicinales. Posteriormente los ejemplares herborizados (Savile, 1962; MBG, 1997) fueron determinados en el Herbario MOL-Augusto Weberbauer de la Universidad Nacional Agraria La Molina y el Herbario USM (Universidad Nacional Mayor de San Marcos).

En paralelo a las caminatas etnobotánicas se realizaron reuniones con los pobladores entrevistados utilizando la técnica del "mapa parlante" (Cox, 1996; Rado, 2011; Hoffmann et al., 2014) para obtener información de sus recursos vegetales, áreas de cultivo y sobre todo el generar un ambiente amical con los pobladores.

\section{Resultados y discusión}

Se determinaron un total de 62 especies de plantas con uso medicinal agrupadas en 47 géneros y 28 familias. Para todas las especies, se reportan su nombre común, uso específico, partes utilizadas y uso 
acompañante (Tabla 1, al final). La familia Asteraceae es el grupo con más representantes usados sumando 22 especies, seguido por Geraniaceae y Urticaceae con 4 especies cada una, Polygonaceae y Rosaceae con 3 especies cada una y las demás con 1 ó 2 especies.

La mayoría de las especies medicinales de Quero se comparten con las usadas en comunidades de la provincia de Canta en Lima (De la Cruz et al., 2007), en las comunidades de Masma Chicche y Quero de Jauja (Siura \& Flores, 2010) y en el valle del Mantaro (Tovar, 2001). Sin embargo, cuatro especies: Cantua buxifolia, Cenchrus clandestinus, Daucus montanus y Descurainia athrocarpa, son novedades con uso medicinal para la región Junín, aunque dos especies sí son utilizadas en otras regiones del Perú. Huamantupa et al. (2011) reporta el uso medicinal de Cantua buxifolia en el Cuzco y es usada por los pobladores como febrífugo, Bussmann \& Sharon (2016) registran a Daucus montanus en el norte del Perú y es usado para el mal de aire, hechicería, torceduras, entre otros. Las otras dos especies no registran uso medicinal fuera de Junín en la actualidad.

La predominancia en el uso de asteráceas tiene sentido, ya que esta familia posee componentes como terpenos (lactonas sesquiterpénicas), señaladas como antinflamatorias, diuréticas y antiespasmódicas (De la Cruz et al., 2007). De la misma forma las urticáceas contienen taninos y sales minerales (Hierro, Calcio, Silicio, Magnesio, Potasio) y sus propiedades irritantes a la piel se debe a su contenido de ácido fórmico, histamina y acetilcolina. El conjunto de estos metabolitos genera propiedades antiinflamatorias, diuréticas, depurativas, hemostáticas y remineralizante (Marrassini et al., 2010). Las caprifoliáceas contienen fenoles, terpenos (Valenos), ácidos esenciales, alcaloides y colina a los cuales se les atribuye propiedades emolientes y ligeramente laxantes, antirreumático, antioxidante, sedante al sistema nervioso central y antiespasmódico (Martínez et al., 2008; Ruiz et al., 2013). Las polygonáceas contienen flavonoides, ácidos fenólicos, taninos, saponinas y esteroles, los cuales tienen acción gastrointestinal (Hernández et al., 2005; Beltrán et al., 2013).

En el Distrito de Quero las plantas medicinales se utilizan para aliviar 12 dolencias (Tabla 2), según la Clasificación Internacional de Enfermedades $10^{\circ}$ - CIE $10^{\circ}$ de la Organización Mundial de la Salud (Mezzich et al., 1995; Christo et al., 2006; Camasca, 2012; Odonne et al., 2013).

En el valle del Mantaro (Tovar, 2001) describió la predominancia de plantas que curan dolencias ligadas al hígado y riñón seguido de males digestivos. Estudios de comercialización de plantas medicinales en Masma Chicche y Quero, ambos ubicados en Jauja-Junín (Puelles et al., 2010), registran una mayor demanda comercial de plantas que alivian enfermedades relacionadas a las vías respiratorias y articulares (reumatismo y otros).
Tabla 2. Categoría de dolencias y número de especies medicinales.

\begin{tabular}{|c|c|c|}
\hline $\mathbf{N}^{\circ}$ & Categoría de dolencias & $\begin{array}{c}\text { Número } \\
\text { de } \\
\text { especies }\end{array}$ \\
\hline 1 & $\begin{array}{l}\text { Traumatismos: golpe, } \\
\text { fractura, quemadura, herida. }\end{array}$ & 18 \\
\hline 2 & Sistema $\quad$ respiratorio: & \\
\hline & $\begin{array}{l}\text { bronquitis, tos, rinitis, } \\
\text { amigdalitis. }\end{array}$ & 17 \\
\hline 3 & $\begin{array}{l}\text { No definidas: susto, colerina } \\
\text { (enfado), frío, bebés que no } \\
\text { hablan. }\end{array}$ & 16 \\
\hline 4 & $\begin{array}{l}\text { Sistema digestivo: gastritis, } \\
\text { ulcera, vesícula. }\end{array}$ & 16 \\
\hline 5 & $\begin{array}{l}\text { Sistema genitourinario: } \\
\text { prostatitis, } \\
\text { urinaria, infección } \\
\text { menstruales, infección } \\
\text { vaginal, inflamación del } \\
\text { ovario, dolor de riñón. }\end{array}$ & 14 \\
\hline 6 & $\begin{array}{l}\text { Infecciosas y parasitarias: } \\
\text { indigestión, cólico } \\
\text { estomacal, disentería. }\end{array}$ & 11 \\
\hline 7 & $\begin{array}{l}\text { Sistema circulatorio: } \\
\text { presión alta, taquicardia. }\end{array}$ & 5 \\
\hline 8 & $\begin{array}{l}\text { Sistema osteomuscular: } \\
\text { reumatismo, dolor } \\
\text { muscular. }\end{array}$ & 4 \\
\hline 9 & $\begin{array}{l}\text { Sangre y de los órganos } \\
\text { hematopoyéticos: anemia, } \\
\text { debilidad. }\end{array}$ & 1 \\
\hline 10 & Sistema nervioso: cefalea & 1 \\
\hline 11 & $\begin{array}{l}\text { Oído y de la apófisis } \\
\text { mastoides: otitis, dolor de } \\
\text { oído. }\end{array}$ & 1 \\
\hline 12 & $\begin{array}{l}\text { Embarazo, parto } \\
\text { puerperio: postparto. }\end{array}$ & 1 \\
\hline
\end{tabular}

Quero se ubica en la ecorregión Puna, cuenta con dos áreas relacionadas a las actividades agrosilvopastoriles. La primera, entre los 3700 a 4000 metros de elevación, es una zona de cultivo cercana a las viviendas, ríos y puquiales. En esta zona se encontraron la mayoría de especies medicinales como: Urtica magellanica "ortiga blanca", Urtica urens "ortiga negra", Caiophora cirsiifolia "ortiga colorada", Achyrocline alata "árnica", Rumex cuneifolius "cuturrumasa", y especies cultivadas por los pobladores como es en el caso de Borago officinalis "borraja", Matricaria chamomilla "manzanilla" y Solanum tuberosum "papa".

La segunda, es la zona alta entre los 3900 a 4800 metros de elevación, con predominancia de pajonales y aprovechado para el pastoreo de camélidos. En esta zona se encontraron especies silvestres como Senecio canescens "wila wila", Perezia pinnatifida "valeriana hembra y valeriana macho" y Werneria villosa "uña cristuma".

Por las entrevistas realizadas a los pobladores se registra una mayor frecuencia de uso de las especies: Caiophora cirsiifolia "ortiga colorada", Borago 
officinalis "borraja", Rumex cuneifolius "cuturrumasa", Matricaria chamomilla "manzanilla". Respecto al manejo de las plantas medicinales; cultivadas o silvestres, 19 personas (83\%) manifestaron no cultivar, mientras que 1 persona (4\%) afirmó que sí, y 3 personas (13\%) no respondieron.

Por otro lado, los pobladores de Quero reconocen a aquellas personas que curan o alivian los males con plantas extraídas del campo y los llaman los "naturistas" y deslindan alguna relación con los chamanes, brujos o curanderos. Los "naturistas" conocen en amplitud a las plantas medicinales de Quero, saben del hábitat de la planta, así como el uso adecuado de sus partes, la preparación y dosis de administración. La actividad de los "naturistas" de Quero contrasta con los llamados "chamanes o curanderos" de la región amazónica de Junín, donde estos son reconocidos como transmisores del poder mágico de las plantas (Arredondo, 2006; Valadeaua et al., 2010; Pardo de Santayana et al., 2011; Odonne et al., 2013), y, en el norte del Perú, contrasta también con los "brujos o curanderos", los cuales utilizan las plantas, en su mayoría "mágicas", para contactarse con los espíritus o realizar rituales de magia (De Feo, 2003; Bussmann et al., 2007a).

Una visión que tienen los pobladores de Quero sobre las plantas medicinales es atribuir sexualidad a las plantas diferenciando masculino "macho" y femenino "hembra" de acuerdo a las características morfológicas que presenta el espécimen. Así, llaman a Sonchus oleraceus "amargón macho" el cual se caracteriza por tener hojas verdes con tonalidades moradas, en contraparte llaman "amargón hembra" a Taraxacum officinale caracterizado por tener solo hojas verdes. En el caso de Perezia pinnatifida los pobladores diferencian dos tipos de nombres comunes en base a diferencias morfológicas; es así que llaman "valeriana hembra" a la planta con hojas pequeñas pinnatilobadas y de borde ciliado, y "valeriana macho" que tiene hojas grandes y ápice espinescente.

Estudios etnobotanicos realizados por Franquemont et al. (1990) en la comunidad de Chincheros (Cuzco), y basados en la cosmovisión andina de los pobladores, señalan a Perezia pinnatifida como "valeriana hembra" y la catalogan como una planta más grande, útil (fértil) y fuerte. Y en el caso de "valeriana macho", a Perezia pungens, por ser débil e infértil. Si bien las sociedades individuales pueden diferir considerablemente en su conceptualización de las plantas y animales, hay una serie de principios estructurales que regulan la clasificación biológica popular que son bastante generales (Berlin et al., 1973).

En las entrevistas también se realizaron preguntas sobre la transmisión del conocimiento de las plantas medicinales, las personas respondieron que la realizan de forma oral, por lo general de los padres a los hijos o familiares. Asimismo, señalaron que los jóvenes de Quero usan las plantas medicinales y al acompañar a los adultos en la recolección son a su vez adiestrados para poder identificar las especies medicinales, así como conocer el uso y la dosis requerida para aliviar algún mal. Sin embargo, muchos de los adolescentes migran a la ciudad de Jauja y Huancayo por trabajo o estudios, pudiéndose producir así la pérdida de la transmisión de conocimientos sobre el uso de las plantas medicinales. En Cuzco y Chachapoyas, son los estudios secundarios motivo de emigración para muchos jóvenes (Malengreau, 2007).

En el norte del Perú, estudios etnobotánicos revelan que el conocimiento de las plantas medicinales todavía se enseña de forma oral, sin constancia escrita. Para Schultes, (1997) y Bussmann (2007a) una guía ilustrada de identificación para las plantas medicinales ayudaría a mantener vivo el conocimiento tradicional.

\section{Conclusiones}

Los pobladores de Quero utilizan y conocen las propiedades medicinales de especies de su flora local y son los "naturistas" las personas con mayor conocimiento. Asimismo, se registró un total de 62 especies, siendo Asteraceae la familia predominante. Las dolencias más frecuentes fueron las relacionadas al sistema genitourinario, sistema digestivo, sistema respiratorio y traumatismos.

Por último, la información obtenida puede servir para futuras investigaciones en temas relacionado a la salud, economía y estudios de mercado, manejo de recursos entre otros.

\section{Agradecimientos}

Un especial agradecimiento a los pobladores de la comunidad de Quero, que contribuyeron proporcionando información valiosa al conocimiento etnobotánico del lugar. A la familia Cristóbal por su apoyo y cordial recepción durante la estadía en la comunidad. A José Alegría y Arturo Granda por su valioso aporte en la revisión del artículo, así como la revisión de algunas especies. Y todas aquellas personas que participaron en esta investigación.

\section{Literatura citada}

Albuquerque U.P., Reinaldo R.C.P., Santiago A.C.P. \& Medeiros P. 2015. Do ferns and lycophytes function as medicinal plants? A study of their low representation in traditional pharmacopoeias. Journal of Ethnopharmacology, 175: 39-47.

Alexiades M.N. 1996. Standard techniques for collecting and preparing herbarium specimens. In: Alexiades M.N. (ed). Selected Guidelines for ethnobotanical research: A field manual. Advances in Economic Botany 10. The New York Botanical Garden, Bronx, New York.

Arredondo F. 2006. Dualidad simbólica de plantas y animales en la práctica médica del curandero-paciente en Huancayo. Tesis Para optar el Grado de Magister en Antropología. Pontificia Universidad Católica Del Perú. 
Beltrán C., Díaz F. \& Gómez H. 2013. Tamizaje fitoquímico preliminar de especies de plantas promisorias de la costa atlántica colombiana. Universidad de Cartagena. Cartagena de Indias, Colombia. Revista Cubana de Plantas Medicinales.

Berlin B., Breedlove D. \& Raven P. 1973. General Principles of Classification and Nomenclature in Folk Biology. American Anthropologist, New Series, 75(1): 214-242.

Bermúdez A., Oliveira-Miranda M. \& Velázquez D. 2005. La investigación etnobotánica sobre plantas medicinales: una revisión de sus objetivos y enfoques actuales. Interciencia, 30(8): 453-459. Caracas.

Blasco T. \& Otero L. 2008. Técnicas conversacionales para la recogida de datos en investigación cualitativa: La entrevista (I). Revista Nure Investigación, $N^{\circ} 33$. Centro Nacional de Medicina Tropical. Instituto de Salud Carlos III.

Brack A. 1999. Diccionario enciclopédico de plantas útiles del Perú. Programa de las Naciones Unidas Para el Desarrollo, Centro Bartolomé de las Casas, Cuzco.

Bussmann R., Sharon D., Vandebroek, I., Jones A. \& Revene Z. 2007a. Health for sale: the medicinal plant markets in Trujillo and Chiclayo, Northern Peru. Journal of Ethnobiology and Ethnomedicine, 3:37.

Bussmann R., Sharon D. \& Lopez A. 2007b. Blending Traditional and Western Medicine: Medicinal plant use among patients at Clinica Anticona in El Porvenir, Peru. Journal Ethnobotany Research \& Applications. 5:185199.

Bussmann R. \& Sharon D. 2016. Plantas Medicinales de los Andes y la Amazonía. La Flora Mágica y Medicinal del Norte del Perú. Centro William L. Brown-Jardín Botánico de Missouri. Trujillo-Perú.

Caballero J., Cortez L., Martínez-Alfaro M. \& Lira-Saade R. 2004. Biodiversidad de Oxaca. Uso y manejo de la diversidad vegetal. 1 ed. Editorial Redacta S.A. DE C.V. México.

Camasca A. 2012. Estudio de la demanda y estimación del Valor Cultural y Económico de plantas medicinales comercializadas en la cuidad de Ayacucho. Tesis para optar al grado académico de magíster en Botánica Tropical con mención en Botánica Económica. Escuela de Posgrado, Facultad de Ciencias Biológicas, Universidad Nacional Mayor de San Marcos, Lima Perú.

Ceuterick M., Vandebroek I. \& Pieroni A. 2011. Resilience of Andean urban ethnobotanies: a comparison of medicinal plant use among Bolivian and Peruvian migrants in the United Kingdom and in their countries of origin. Journal of Ethnopharmacology, 136: 27-54

Christo A., Guedes-Bruni R. \& Fonseca-Kruel V.S. da V. 2006. Uso de Recursos vegetais em comunidades rurais Limítrofes à Reserva Biológica de Poço das Antas, Silva Jardim, Rio de Janeiro: Estudo de caso na Gleba Aldeia Velha. Rodriguésia, 57: 519-542.

Cox R. 1996. El saber local, metodologías y técnicas participativas. NOGUB-COSUDE /CAF. La Paz, Bolivia.

De Feo V. 2003. Ethnomedical field study in northern Peruvian Andes with particular reference to divination practices. Journal of Ethnopharmacology, 85: 243-256.
De La Torre L., Muriel P. \& Balslev H. 2006. Etnobotánica en los Andes del Ecuador. Botánica Económica de los Andes Centrales, 246-267. Universidad Mayor de San Andrés, La Paz. Quito. Ecuador.

De la Cruz H., Vilcapoma G. \& Zevallos P. 2007. Ethnobotanical study of medicinal plants used by the Andean people of Canta, Lima, Peru. Journal of Ethnopharmacology, 111: 284-294.

Franquemont C., Plowman T., Franquemont E., King S.R., Niezgoda C., Davis W. \& Sperling C.R. 1990. Ethnobotany of Chinchero, an Andean Community in Southern Peru. In: Fieldiana-Botany; New Series, N-24. Publication 1408. Published by Field Museum of Natural History.

Gómez V. \& Vilca P. 2006. Efectos Positivos y negativos de la liberalización de los mercados en la economía de las comunidades campesinas de la sierra del Perú: Caso de la Comunidad Campesina de Quero.

Hernández T., Canales M. \& Caballero J. 2005. Análisis Cuantitativo del Conocimiento Tradicional Sobre Plantas Utilizadas Para el Tratamiento de Enfermedades Gastrointestinales en Zapotitlán de las Salinas, Puebla, México. Revista Interciencia, 30(9): 529-535.

Hoffmann D., Tarquino R., Corro J.F. \& Lavadenz L. 2014. Métodos para caracterizar la dinámica de los sistemas socioecológicos asociados a los bofedales altoandinos (Cordillera Real, Bolivia). Ecología en Bolivia, 49(3): 132-140.

Horák M., Somerlíková K., Kavenská V., Granda L., Škrabáková L., Tournon J. Chuspe M., Rosero M., Forero L., Rosero A., Castro N., Beltrán G., Halbich M., Minero F., Rosas I. \& Mateos E. 2015. Etnobotánica y fitoterapia en América. Horák M. (Editor). Universidad de Mendel en Brno, Facultad de Desarollo Regional y Estudios Internacionales, Departamento de Idiomas y Estudios Culturales. República Checa.

Huamantupa I., Cuba M., Urrunaga R., Paz E., Ananya N., Callalli M., Pallqui N. \& Coasaca H. 2011. Riqueza, uso $\mathrm{y}$ origen de plantas medicinales expendidas en los mercados de la ciudad del Cusco. Facultad de Ciencias Biologicas UNMSM. Rev. Peru. Biol. 18(3): 283-291. DOI: https://doi.org/10.15381/rpb.v18i3.439.

La Torre-Cuadros M. \& Albán J. 2006. Etnobotánica en los Andes del Perú. Botánica Económica de los Andes Centrales, 239-245. Universidad Mayor de San Andrés, La Paz., Bolivia.

Malengreau J. 2007. Migraciones entre lo local y lo regional en los Andes peruanos: redes rural-urbanas, fragmentaciones espaciales y recomposiciones identitarias. Bulletin de l'Institut Français d'Études Andines, 36(3): 427-445.

Marrassini C., Gorzalczany S. \& Ferraro G. 2010. Actividad analgésica de dos especies de Urtica con usos etnomédicos en la República Argentina. Dominguezia, $6(1)$.

Martínez A., Valencia G., Jiménez N., Mesa M. \& Galeano E. 2008. Manual de Prácticas de Laboratorio de Farmacognosia y Fitoquímica. Universidad de Antioquia. Facultad de Química Farmacéutica. Departamento de Farmacia. Medellín, Colombia.

Martínez C. 2011. El muestreo en investigación cualitativa. Principios básicos y algunas controversias. Departamento de Atención a la Salud, Universidad Autónoma Metropolitana (Xochimilco). DF México. 
Mezzich J.E., Zapata M. \& Schwartz K. 1995. La Nueva Clasificación Internacional de Enfermedades y el Diagnóstico Multiaxial en Medicina General. Revista Médica Herediana, 6(4).

MBG (Missouri Botanical Garden). 1997. Técnicas De Campo Utilizadas Por El Jardín Botánico De Missouri.

Odonne G., Valadeau C., Alban-Castillo J., Stien D., Sauvain M. \& Bourdy G. 2013. Medical ethnobotany of the Chayahuita of the Paranapura basin (Peruvian Amazon). Journal of Ethnopharmacology, 146:127-153.

OMS. 2003. Medicina Tradicional. Informe de la Secretaría. Organización Mundial de la Salud. 56 a ASAMBLEA MUNDIAL DE LA SALUD. Punto 14.10 del orden del día provisional.

Pardo de Santayana M., Jaureguia X., Clavo Z.M. \& Jovelc E.M. 2011. "Plantas con madre": Plants that teach and guide in the shamanic initiation process in the EastCentral Peruvian Amazon. Journal of Ethnopharmacology, 134: 739-752.

Puelles M., Gómez V., Moris J., de Felipe I., Briz J., Siura S., Briz T., Flores M. \& Pérez M. (Eds). 2010. Las plantas medicinales de Perú. Etnobotánica y viabilidad comercial. Universidad Complutense De Madrid; Isabel De Felipe Boente, Universidad Politécnica De Madrid. Los Libros De La Catarata.

Rado B. 2011. Etnobotánica del Distrito de Ocongate Quispicanchi - Cusco. Universidad Nacional San Antonio Abad del Cusco. URI http://repositorio.unsaac.edu.pe/handle/UNSAAC/827.

Revene Z., Bussmann R. \& Sharon D. 2008. From Sierra to Coast: Tracing the supply of medicinal plants in Northern Peru - A plant collector's tale. Ethnobotany Research and Applications, 6: 15-22.
Ruiz S., Venegas E. \& Ruidías D. 2013. Capacidad Antioxidante in vitro de los Flavonoides Totales Obtenidos de las Hojas de Sambucus peruviana H.B.K. (Sauco) Proveniente de la Ciudad de Huamachuco. Revista Pharmaciencia, 1(2): 57-64. URI: http://revistas.unitru.edu.pe/index.php/farmabioq/article/ view/464/419.

Savile D.B.O. 1962. Collection and Care of Botanical Specimens. Reprinted with Addenda 1973. Publication 1113. Research Branch, Canada Department of Agriculture.

Schultes RE. 1997. The Importance of Ethnobotany Environmental Conservation. Monograf Jard. Bot. Córdova, 5: 157-164. Harvard University. MS. USA.

Siura S. \& Flores M. 2010. Las plantas medicinales de Perú. Etnobotánica de las Plantas Medicinales de las Comunidades Campesinas de Quero y Masma Chicche. 33-48.

Sõukand R. \& Kalle R. 2010. Herbal Landscape: the perception of the landscape as a source of medicinal plants. Trames, 14(64/59): 207-226.

Tovar O. 2001. Plantas Medicinales Del Valle Del Mantaro. Museo de Historia Natural. Universidad San Marcos. Multicopy, 7: 13-17.

Valadeaua C., Alban J., Sauvain M., Lores A.F. \& Bourdy G. 2010. The rainbow hurts my skin: Medicinal concepts and plants uses among the Yanesha (Amuesha), an Amazonian Peruvian ethnic group. Journal of Ethnopharmacology, 127(1): 175-192. DOI: 10.1016/j.jep.2009.09.024.

Weckerle C., Bussmann R.W. \& Monigatti M. 2013. Medicinal plant use in two Andean communities located at different altitudes in the Bolívar Province, Peru. Journal of Ethnopharmacology, 145: 450-464.

Zeni A.L.B., Tribess B., Pintarelli G.M., Bini L.A., Camargo A., Funez L. \& De Gasper A.L. 2015. Ethnobotanical study of plants used for therapeutic purposes in the Atlantic Forest region, Southern Brazil. Journal of Ethnopharmacology, 164: 136-146.

Tabla 1. Plantas medicinales de la comunidad de Quero.

\begin{tabular}{|c|c|c|c|c|}
\hline Nombre Científico & Nombre Común & USO & $\begin{array}{l}\text { Uso / Parte } \\
\text { utilizada }\end{array}$ & Uso acompañado \\
\hline \multicolumn{5}{|l|}{ Adoxaceae } \\
\hline Sambucus peruviana Kunth & yalán o sauco & $\begin{array}{l}\text { Bronquios, } \\
\text { heridas, } \\
\text { reumatismo. }\end{array}$ & Hojas, tallo. & Ninguno \\
\hline \multicolumn{5}{|l|}{ Apiaceae } \\
\hline Daucus montanus Humb. \& Bonpl. ex Schult. & pachacomino & $\begin{array}{l}\text { Indigestión, } \\
\text { empacho, } \\
\text { estomago flojo }\end{array}$ & $\begin{array}{l}\text { Tallo fresco o } \\
\text { seco. }\end{array}$ & Ninguno \\
\hline \multicolumn{5}{|l|}{ Asteraceae } \\
\hline Achyrocline alata (Kunth) DC. & árnica & $\begin{array}{l}\text { Golpe, rotura, } \\
\text { herida. }\end{array}$ & Hojas, flores. & $\begin{array}{l}\text { Chupasangre, verbena } \\
\text { y chuculucuy. Orine, } \\
\text { perejil, yawarshujo. }\end{array}$ \\
\hline Artemisia absinthium L. & ajenco & Cólico & Hojas & Ninguno \\
\hline Baccharis latifolia (Ruiz \& Pav.) Pers. & chilca & $\begin{array}{l}\text { Rotura, dolores } \\
\text { musculares }\end{array}$ & Hojas & $\begin{array}{l}\text { Árnica, grasa de } \\
\text { animal. }\end{array}$ \\
\hline Chuquiraga spinosa Less. & huamanpinta & $\begin{array}{l}\text { Riñones, } \\
\text { infección } \\
\text { urinaria, } \\
\text { vesícula, } \\
\text { próstata }\end{array}$ & $\begin{array}{l}\text { Hojas, flores, } \\
\text { tallo. }\end{array}$ & $\begin{array}{l}\text { Llantén, cebada } \\
\text { tostada. }\end{array}$ \\
\hline
\end{tabular}


Tabla 1. Plantas medicinales de la comunidad de Quero.

\begin{tabular}{|c|c|c|c|c|}
\hline Nombre Científico & Nombre Común & USO & $\begin{array}{l}\text { Uso / Parte } \\
\text { utilizada }\end{array}$ & Uso acompañado \\
\hline Gamochaeta coarctata (Willd.) Kerguélen & lengua de perro & $\begin{array}{l}\text { Infección } \\
\text { vaginal e } \\
\text { intestinal, } \\
\text { hígado, ulcera, } \\
\text { heridas, } \\
\text { quemaduras }\end{array}$ & Hojas. & $\begin{array}{l}\text { Acashquiro, verbena, } \\
\text { llantén, cuturrumasa }\end{array}$ \\
\hline Leucanthemum maximum (Ramond) DC. & margarita & Roturas & Hojas, flores. & Shuculucuy (lagartija) \\
\hline Matricaria chamomilla $\mathrm{L}$. & manzanilla & $\begin{array}{l}\text { Dolor de } \\
\text { cabeza, } \\
\text { infección } \\
\text { urinario o } \\
\text { inflamación de } \\
\text { ovarios }\end{array}$ & Flor, hojas. & Ninguno \\
\hline Perezia multiflora (Humb. \& Bonpl.) Less. & $\begin{array}{l}\text { escorzonera, } \\
\text { discorsonera }\end{array}$ & $\begin{array}{l}\text { Presión, } \\
\text { bronquios, } \\
\text { gripe. }\end{array}$ & Hojas. & $\begin{array}{l}\text { Ortiga colorada, } \\
\text { llancahuasha, wila } \\
\text { wila. }\end{array}$ \\
\hline Perezia pinnatifida (Humb. \& Bonpl.) Wedd. & $\begin{array}{l}\text { valeriana macho, } \\
\text { raíz valeriana } \\
\text { valeriana hembra }\end{array}$ & $\begin{array}{l}\text { Susto, nervio, } \\
\text { colerina } \\
\text { Susto, colerina, } \\
\text { nervios. }\end{array}$ & $\begin{array}{l}\text { Hojas, flores, } \\
\text { raíz. } \\
\text { Hojas, flor, } \\
\text { raíz. }\end{array}$ & $\begin{array}{l}\text { Choclo, cáscara de } \\
\text { granada. } \\
\text { Ninguno }\end{array}$ \\
\hline Senecio canescens (Humb. \& Bonpl.) Cuatrec. & wila wila & $\begin{array}{l}\text { Bronquios, } \\
\text { infección a la } \\
\text { vía respiratoria, } \\
\text { frío. }\end{array}$ & Hojas, flor & $\begin{array}{l}\text { Ortiga colorada, raíz } \\
\text { valeriana. }\end{array}$ \\
\hline Senecio collinus DC. & jarhuancho & $\begin{array}{l}\text { Susto, } \\
\text { bronquios, frio. }\end{array}$ & Hojas & Ninguno \\
\hline Senecio hohenackeri $\quad$ Sch. Bip. & shushulohuaita & $\begin{array}{l}\text { Inflamación de } \\
\text { ovario, bebes } \\
\text { que no hablan }\end{array}$ & Hojas, flores. & Clavel, pacte. \\
\hline Senecio rhizomatus Rusby & llancahuasha & $\begin{array}{l}\text { Herida, gripe, } \\
\text { inflamación de } \\
\text { ovario, cólicos } \\
\text { menstruales. }\end{array}$ & Flores, hojas. & $\begin{array}{l}\text { Wila wila, ortiga } \\
\text { colorada para el frio. }\end{array}$ \\
\hline Senecio tephrosioides Turcz. & anguiripa & $\begin{array}{l}\text { Tos, bronquios } \\
\text { y gripe. }\end{array}$ & Hojas & $\begin{array}{l}\text { Llancahuasha, ortiga } \\
\text { colorada. }\end{array}$ \\
\hline Senecio timidus Cuatrec & huamanripa & $\begin{array}{l}\text { Gripe, } \\
\text { bronquios. }\end{array}$ & Hojas. & Manzanilla \\
\hline Sonchus oleraceus (L.) L. & amargón macho & Vesícula & Hojas. & Ninguno \\
\hline Tanacetum parthenium (L) Sch. Bip. & santa mata & Maldad & Hojas. & Ninguno \\
\hline $\begin{array}{l}\text { Taraxacum officinale (L.) Weber ex } \\
\text { F.H.Wigg. }\end{array}$ & amargón hembra & $\begin{array}{l}\text { Dolencia al } \\
\text { hígado }\end{array}$ & Hojas. & $\begin{array}{l}\text { Algunas frutas para el } \\
\text { jugo. }\end{array}$ \\
\hline Werneria nubigena Kunth & mamai millai & $\begin{array}{l}\text { Estimula al } \\
\text { apetito }\end{array}$ & Flores, hojas & Ninguno \\
\hline Werneria villosa A. Gray & uña cristuma & $\begin{array}{l}\text { Genera apetito, } \\
\text { inflamación de } \\
\text { ovario y } \\
\text { descenso } \\
\text { blanco. }\end{array}$ & Hojas, flores. & Ninguno \\
\hline $\begin{array}{l}\text { Xenophyllum dactylophyllum (Sch. Bip.) V.A. } \\
\text { Funk }\end{array}$ & cuno cuno & $\begin{array}{l}\text { Cura la tos, } \\
\text { gripe. }\end{array}$ & Hojas & Ninguno \\
\hline \multicolumn{5}{|l|}{ Boraginaceae } \\
\hline Borago officinalis L. & borraja & $\begin{array}{l}\text { Colerina, } \\
\text { nervios, } \\
\text { infección } \\
\text { intestinal, gripe. }\end{array}$ & Hojas, flores. & Ninguno \\
\hline \multicolumn{5}{|l|}{ Brassicaceae } \\
\hline $\begin{array}{l}\text { Descurainia athrocarpa (A. Gray) O.E. } \\
\text { Schulz. } \\
\text { Buddlejaceae }\end{array}$ & sin nombre común & Frío & Hojas. & Ninguno \\
\hline Buddleja coriacea Remy & quishuar & $\begin{array}{l}\text { Resfrío, } \\
\text { bronquios, }\end{array}$ & Hojas, flores. & Ninguno \\
\hline
\end{tabular}


Tabla 1. Plantas medicinales de la comunidad de Quero.

\begin{tabular}{|c|c|c|c|c|}
\hline Nombre Científico & Nombre Común & USO & $\begin{array}{l}\text { Uso / Parte } \\
\text { utilizada }\end{array}$ & Uso acompañado \\
\hline & & $\begin{array}{l}\text { cicatrizante y } \\
\text { hongos. }\end{array}$ & & \\
\hline $\begin{array}{l}\text { Buddleja incana Ruiz \& Pav. } \\
\text { Cactaceae }\end{array}$ & sin nombre común & Frio & Hojas, flores. & Ninguno \\
\hline $\begin{array}{l}\text { Austrocylindropuntia floccosa (Salm-Dyck ex } \\
\text { Winterf) F. Ritter }\end{array}$ & walajo o abuelito & Gastritis & Tallo & $\begin{array}{l}\text { Sabia obtenida del } \\
\text { cladodio de tuna }\end{array}$ \\
\hline \multicolumn{5}{|l|}{ Calceolariaceae } \\
\hline Calceolaria scabra Ruiz \& Pav. & $\begin{array}{l}\text { zapatito, bolsa de } \\
\text { pastor }\end{array}$ & $\begin{array}{l}\text { Inflamación de } \\
\text { ovario y } \\
\text { próstata, mal del } \\
\text { riñón. }\end{array}$ & Hojas y flores & $\begin{array}{l}\text { Papa rayada, cebada, } \\
\text { huamanpinta. }\end{array}$ \\
\hline \multicolumn{5}{|l|}{ Caprifoliaceae } \\
\hline Valeriana connata Ruiz \& Pav. & torpo torpo & $\begin{array}{l}\text { Bronquios, } \\
\text { inflamación de } \\
\text { hígado y } \\
\text { ovarios. }\end{array}$ & Raíz, hojas. & Ninguno \\
\hline Valeriana micropterina Wedd. & huaynacuri & $\begin{array}{l}\text { Nervio, susto, } \\
\text { mal del corazón }\end{array}$ & $\begin{array}{l}\text { Flores, hojas, } \\
\text { raíz. }\end{array}$ & Ninguno \\
\hline \multicolumn{5}{|l|}{ Equisetaceae } \\
\hline Equisetum bogotense Kunth & cola de caballo & $\begin{array}{l}\text { Riñones, heridas } \\
\text { (hemorragia) }\end{array}$ & Tallo & $\begin{array}{l}\text { Cebada tostada, } \\
\text { llantén, cuturrumasa. }\end{array}$ \\
\hline \multicolumn{5}{|l|}{ Fabaceae } \\
\hline $\begin{array}{l}\text { Senna versicolor (Vogel) H.S. Irwin \& } \\
\text { Barneby }\end{array}$ & pacte & $\begin{array}{l}\text { Susto, } \\
\text { inflamación } \\
\text { intestinal. }\end{array}$ & Hojas, flores & $\begin{array}{l}\text { Orine y jabón negro } \\
\text { para la inflamación } \\
\text { intestinal. Flores de } \\
\text { clavel, rosa, retama } \\
\text { para el susto. }\end{array}$ \\
\hline \multicolumn{5}{|l|}{ Gentianaceae } \\
\hline $\begin{array}{l}\text { Gentianella aff. scarlatinostriata (Gilg) } \\
\text { Zarucchi } \\
\text { Geraniaceae }\end{array}$ & sumayshunsho & Dolor de muela & $\begin{array}{l}\text { Flor, hojas, } \\
\text { raíz. }\end{array}$ & Ninguno \\
\hline Erodium cicutarium (L.) L'Hér. & aspadilla & Colerina & Hojas & Ninguno \\
\hline Erodium moschatum (L.) L'Hér. & auja auja & $\begin{array}{l}\text { Infección } \\
\text { estomacal, } \\
\text { colerina. }\end{array}$ & Hojas. & $\begin{array}{l}\text { Pachamuña y } 5 \text { heces } \\
\text { tostadas de cuy. }\end{array}$ \\
\hline Geranium sessiliflorum Cav. & ahuachilla & $\begin{array}{l}\text { Golpe, rotura, } \\
\text { herida. }\end{array}$ & & Chupasangre \\
\hline Pelargonium $\times$ hortorum L.H. Bailey & geranio & $\begin{array}{l}\text { Inflamación de } \\
\text { garganta o dolor } \\
\text { de amígdalas }\end{array}$ & Hojas. & Ninguno \\
\hline \multicolumn{5}{|l|}{ Lamiaceae } \\
\hline Minthostachys mollis (Benth.) Griseb. & pacha muña & $\begin{array}{l}\text { Dolor de } \\
\text { barriga, diarrea } \\
\text { con sangre, } \\
\text { gastritis. }\end{array}$ & Hojas, flores & Ninguno \\
\hline \multicolumn{5}{|l|}{ Loasaceae } \\
\hline Caiophora cirsiifolia C. Presl & ortiga colorada & $\begin{array}{l}\text { Gripe, } \\
\text { bronquios, } \\
\text { resfrío, tos, } \\
\text { quitar el frío. }\end{array}$ & Hojas. & $\begin{array}{l}\text { Borraja y } \\
\text { escorzonera. }\end{array}$ \\
\hline \multicolumn{5}{|l|}{ Malvaceae } \\
\hline Acaulimalva rhizantha (A.Gray) Krapov. & raíz artia & $\begin{array}{l}\text { Infección de } \\
\text { riñones, } \\
\text { inflamación de } \\
\text { ovario, hígado. }\end{array}$ & Raíz. & $\begin{array}{l}\text { Cebada tostada, } \\
\text { llantén y cola de } \\
\text { caballo. }\end{array}$ \\
\hline \multicolumn{5}{|l|}{ Myrtaceae } \\
\hline Eucalyptus globulus Labill. & eucalipto & $\begin{array}{l}\text { Nervios, tos, } \\
\text { asma, bronquios }\end{array}$ & Hojas jóvenes. & Ninguno \\
\hline \multicolumn{5}{|l|}{ Onagraceae } \\
\hline Oenothera multicaulis Ruiz \& Pav. & $\begin{array}{l}\text { chupasangre, } \\
\text { yawarshujo }\end{array}$ & Heridas, golpes. & Hojas. & Awachilla \\
\hline
\end{tabular}


Tabla 1. Plantas medicinales de la comunidad de Quero.

\begin{tabular}{|c|c|c|c|c|}
\hline Nombre Científico & Nombre Común & USO & $\begin{array}{l}\text { Uso / Parte } \\
\text { utilizada }\end{array}$ & Uso acompañado \\
\hline \multicolumn{5}{|l|}{ Phrymaceae } \\
\hline Mimulus glabratus Kunth & berro amarillo & $\begin{array}{l}\text { Vesícula e } \\
\text { hígado. }\end{array}$ & Hojas. & Ninguno \\
\hline \multicolumn{5}{|l|}{ Plantaginaceae } \\
\hline Plantago australis Lam. & llantén & & Hojas. & Cebada tostada. \\
\hline Veronica anagallis-aquatica $\mathrm{L}$. & hoja de chilco & $\begin{array}{l}\text { Cólicos, } \\
\text { gastritis. }\end{array}$ & Hojas. & $\begin{array}{l}\text { Hojas de apio y jugo } \\
\text { de limón. }\end{array}$ \\
\hline \multicolumn{5}{|l|}{ Poaceae } \\
\hline $\begin{array}{l}\text { Cenchrus clandestinus (Hochst. ex Chiov.) } \\
\text { Morrone }\end{array}$ & grama & Debilidad & $\begin{array}{l}\text { Raíz, tallo, } \\
\text { hojas. }\end{array}$ & $\begin{array}{l}\text { Cebada tostada, } \\
\text { cascaras de papa } \\
\text { blanca. }\end{array}$ \\
\hline \multicolumn{5}{|l|}{ Polemoniaceae } \\
\hline $\begin{array}{l}\text { Cantua buxifolia Juss. ex Lam. } \\
\text { Polygonaceae }\end{array}$ & cantuta & Amígdalas & Flores. & Ninguno \\
\hline Muehlenbeckia volcanica (Benth.) Endl. & mullaca & Bronquios & Raíz. & Ninguno \\
\hline Rumex cuneifolius Campd. & cuturrumasa & $\begin{array}{l}\text { Infección } \\
\text { estomacal, } \\
\text { cicatriza heridas } \\
\text { de operaciones o } \\
\text { heridas internas. }\end{array}$ & Hojas. & Llantén (heridas) \\
\hline \multicolumn{5}{|l|}{ Rosaceae } \\
\hline Acaena ovalifolia Ruiz \& Pav. & shumpo, shurpo & $\begin{array}{l}\text { Gastritis, } \\
\text { hígado. }\end{array}$ & $\begin{array}{l}\text { Tallo, hojas, } \\
\text { flores. }\end{array}$ & Ninguno \\
\hline Lachemilla bipinnatifida (L.M. Perry) Rothm. & acashquiro & $\begin{array}{l}\text { Infección } \\
\text { intestinal o } \\
\text { vaginal, mal de } \\
\text { hígado, colerina }\end{array}$ & $\begin{array}{l}\text { Hojas, raíz, } \\
\text { tallo, flores. }\end{array}$ & Ninguno \\
\hline Sanguisorba minor Scop. & pimpinela & Susto & Hojas. & Toronjil \\
\hline $\begin{array}{l}\text { Solanaceae } \\
\text { Solanum aloysiifolium Dunal }\end{array}$ & mancapachia & Presión alta & $\begin{array}{l}\text { Hojas, flores, } \\
\text { fruto }\end{array}$ & Ninguno \\
\hline \multirow[t]{3}{*}{ Solanum tuberosum $\mathrm{L}$. } & papa blanca & $\begin{array}{l}\text { Colerina, } \\
\text { presión, riñón. }\end{array}$ & Tubérculo & $\begin{array}{l}\text { Ortiga, cola de } \\
\text { caballo, cebada, raíz } \\
\text { artia. }\end{array}$ \\
\hline & papa callhuash & Presión & Tubérculo & Ninguno \\
\hline & papa shiri & Gastritis & Tubérculo & Ninguno \\
\hline \multicolumn{5}{|l|}{ Tropaeolaceae } \\
\hline \multicolumn{5}{|l|}{ Ruiz \& Pav. } \\
\hline \multicolumn{5}{|l|}{ Urticaceae } \\
\hline Urtica echinata Benth. & ortiga vintosiñaule & $\begin{array}{l}\text { Resfrío, dolor } \\
\text { de oído y frio. }\end{array}$ & Hojas. & Ninguno \\
\hline Urtica flabellata Kunth & $\begin{array}{l}\text { ortiga } \\
\text { mulahuañuchi, } \\
\text { mataburro, } 24 \\
\text { horas, ortiga } \\
\text { crespa. }\end{array}$ & $\begin{array}{l}\text { Frio, } \\
\text { reumatismo. }\end{array}$ & Hojas. & Ninguno \\
\hline Urtica magellanica Juss. ex Poir. & ortiga negra & $\begin{array}{l}\text { Frio, calambre y } \\
\text { colerina. }\end{array}$ & Hojas. & Emoliente \\
\hline Urtica urens $\mathrm{L}$. & ortiga blanca & $\begin{array}{l}\text { Resfrío, } \\
\text { bronquios, tos, } \\
\text { dolor de cabeza. } \\
\text { Hemorragia en } \\
\text { general, colerina } \\
\text { o sobreparto. }\end{array}$ & $\begin{array}{l}\text { Hojas, flores, } \\
\text { raíz. }\end{array}$ & Ninguno \\
\hline \multicolumn{5}{|l|}{ Verbenaceae } \\
\hline Verbena litoralis Kunth & verbena & $\begin{array}{l}\text { Infección, } \\
\text { hígado. }\end{array}$ & Hojas, flores. & Ninguno \\
\hline
\end{tabular}


Apéndice 1. Entrevista semiestructurada para determinar conocimiento sobre el uso de las plantas medicinales.

Nombre del encuestado:

Comunidad:

Lugar de nacimiento:

Edad:

Estado Civil

Sexo:

Tiempo en la comunidad:

Observaciones:

1. ¿En su comunidad existen curanderos? ¿Quiénes son?

2. ¿Usan frecuentemente plantas medicinales? ¿En qué ocasiones?

3. ¿En qué ocasiones usan medicamentos?

4. ¿Cuándo las personas se enferman, primero acuden a un curandero o la posta médica? ¿Por qué?

5. ¿Conoces sobre el uso de las plantas medicinales? ¿Quién te enseñó ese conocimiento?

6. ¿Les enseñas a tus hijos el conocimiento sobre las plantas medicinales?

7. ¿Siembras plantas medicinales? ¿Cómo lo haces?

8. ¿Son abundantes o escasas las plantas medicinales de tu comunidad en la actualidad?

9. ¿Por qué crees que existe esa cantidad en la actualidad?

10. Observaciones y comentarios

\footnotetext{
${ }^{1}$ Universidad Nacional Agraria La Molina. Av. La Molina s/n. Lima. Perú. tcgladys@gmail.com.

${ }^{2}$ Universidad Nacional Agraria La Molina. Av. La Molina s/n. Lima. Perú.
} 